\title{
BMJ Open Introducing structured caregiver training in stroke care: findings from the TRACS process evaluation study
}

\author{
David J Clarke, ${ }^{1}$ R Hawkins, ${ }^{2}$ E Sadler, ${ }^{3}$ G Harding, ${ }^{4}$ C McKevitt, ${ }^{3}$ M Godfrey, ${ }^{2}$ \\ J Dickerson, ${ }^{1}$ A J Farrin, ${ }^{5}$ L Kalra, ${ }^{6}$ D Smithard, ${ }^{7}$ A Forster ${ }^{1}$
}

To cite: Clarke DJ,

Hawkins R, Sadler E, et al. Introducing structured caregiver training in stroke care: findings from the TRACS process evaluation study. BMJ Open 2014;4: e004473. doi:10.1136/ bmjopen-2013-004473

- Prepublication history and additional material for this paper is available. To view please visit the journal (http://dx.doi.org/10.1136/ bmjopen-2013-004473).

Received 13 November 2013 Revised 14 March 2014 Accepted 23 March 2014

For numbered affiliations see end of article.

\section{Correspondence to} Dr David J Clarke; d.j.clarke@leeds.ac.uk

\section{ABSTRACT}

Objective: To evaluate the process of implementation of the modified London Stroke Carers Training Course (LSCTC) in the Training Caregivers After Stroke

(TRACS) cluster randomised trial and contribute to the interpretation of the TRACS trial results. The LSCTC was a structured competency-based training programme designed to help develop the knowledge and skills (eg, patient handling or transfer skills) essential for the day-to-day management of disabled survivors of stroke. The LSCTC comprised 14 components, 6 were mandatory (and delivered to all) and 8 non-mandatory, to be delivered based on individual assessment of caregiver need.

Design: Process evaluation using non-participant observation, documentary analysis and semistructured interviews.

Participants: Patients with stroke $(n=38)$, caregivers $(n=38)$, stroke unit staff $(n=53)$.

Settings: 10 of the 36 stroke units participating in the TRACS trial in four English regions (Yorkshire, North West, South East and South West, Peninsula).

Results: Preparatory cascade training on delivery of the LSCTC did not reach all staff and did not lead to multidisciplinary team (MDT) wide understanding of, engagement with or commitment to the LSCTC. Although senior therapists in most intervention units observed developed ownership of the LSCTC, MDT working led to separation rather than integration of delivery of LSCTC elements. Organisational features of stroke units and professionals' patient-focused practices limited the involvement of caregivers. Caregivers were often invited to observe therapy or care being provided by professionals but had few opportunities to make sense of, or to develop knowledge and stroke-specific skills provided by the LSCTC. Where provided, caregiver training came very late in the inpatient stay. Assessment and development of caregiver competence was not commonly observed.

Conclusions: Contextual factors including service improvement pressures and staff perceptions of the necessity for and work required in caregiver training impacted negatively on implementation of the caregiver training intervention. Structured caregiver training programmes such as the LSCTC are unlikely to be practical in settings with short inpatient stays. Stroke units where early supported discharge is in place

\section{Strengths and limitations of this study}

- A major strength of this study was to demonstrate the value of a comprehensive qualitative process evaluation in generating rich and detailed findings which helped to understand and interpret the results of a large pragmatic cluster randomised controlled trial of a poststroke competency based caregiver training intervention which was reported to have no benefit over usual care.

- The process evaluation is one of the largest conducted alongside a stroke rehabilitation trial, with over $12 \mathrm{~h}$ of observation and 92 interviews.

- Another strength of the study was the use of non-participant observations over a period of 6 months. This approach enabled researchers to directly observe the day-to-day rehabilitation work of stroke unit (SU) teams and develop an in-depth understanding of contextual, organisational and professional factors influencing introduction, implementation and embedding of complex interventions into these and similar healthcare settings.

- A limitation of the study is that non-participant observations of practice in participating SUs began almost 1 year after recruitment to the linked pragmatic cluster randomised controlled trial started. Early implementation strategies and staff responses to these could not be directly observed; analysis therefore drew on interview data for this period.

- A further limitation of the study was that only 6 of 18 intervention, and 4 control units were observed. These factors impact on the transferability of the findings to other SUs to some degree.

potentially offer a more effective vehicle for introducing competency based caregiver training.

LINKED TRACS Cluster randomised controlled trial number: ISRCTN49208824.

\section{INTRODUCTION}

Stroke has a profound effect on the lives of patients, their spouses and family members. 
These effects include role and relationship changes, psychological distress and the challenge of coping with long-term disability. ${ }^{1}{ }^{2}$ Family carers provide most postdischarge care to patients but often receive little preparation for this. Long-term caring can result in increased stress and burden for caregivers. ${ }^{1-3}$ A single-centre individually randomised trial reported that the London Stroke Carers Training Course (LSCTC), a structured competency-based training programme, decreased caregiver burden and anxiety, and improved psychological outcomes for patients. Overall costs were reduced, due largely to earlier discharge in the LSCTC group compared with usual care. ${ }^{3} 4$ The pragmatic multicentre cluster randomised controlled trial (Training Caregivers after Stroke, TRACS) ${ }^{5-7}$ was conducted to determine whether the LSCTC ${ }^{3}$ improved patients' functional independence and reduced caregiver burden when delivered as part of routine care.

The LSCTC comprised a 14-item programme delivered by multidisciplinary teams (MDT) during the inpatient stay in stroke units (SUs). The trial was pragmatic, there was no specific time or order for delivery of the LSCTC. Box 1 identifies the 14 LSCTC items, 6 were mandatory, for example, providing information on the nature of stroke, and the importance of medication adherence. Assessment of caregiver need by any member of the MDT determined which non-mandatory items were introduced, for example, providing nutritional support for patients with swallowing difficulties. MDT members were to provide individual training, then assess and record caregiver competence using a standardised training record (see online supplementary file 1). Competence was defined as: the carer has taken on board the knowledge/ skills required to be able to deliver the support the patient needs. The intervention was completed after a follow-up visit or phone call to help caregivers adapt knowledge and skills taught, to the home. Intervention and TRACS trial details are published elsewhere. ${ }^{6}{ }^{7}$ SUs in the control arm provided usual care based on National Clinical Guidelines. ${ }^{7}$ Control unit staff were asked to $\log$ time spent with caregivers but not required to describe the nature of the contact. Compliance by MDT members with delivery of the LSCTC as measured by training record completion was variable (range $0-93 \%$ ).

The outcomes reported in the single centre trial ${ }^{3} 4$ were not replicated in TRACS, which found no clinical or statistical improvement at 6 months between groups on primary outcomes of functional independence (patients), or caregiver burden or for the secondary outcomes measured at 6 and 12 months. ${ }^{6} 7$

Medical Research Council (MRC) Guidelines ${ }^{8}$ recommend process evaluations ${ }^{9}$ to help understand and interpret outcomes of trials of complex interventions such as the LSCTC. We report on a separately funded process evaluation $^{9}$ conducted by researchers independent of the TRACS trial. The process evaluation aimed to: comprehend the context in which formal (LSCTC) or informal caregiver training was implemented in intervention or control SUs

\section{Box 1 LSCTC training components}

The caregiver has demonstrated a knowledge and understanding of

1. His/her relative having had a stroke (mandatory)

2. What a stroke is (mandatory)

3. His/her relative's specific stroke related problems. Possible incomplete recovery and residual unresolved problems
A. Communication and reading
B. Cognition
C. Personality and mood changes
D. Diet and swallowing
E. Vision
F. Personal Activities of Daily Living (PADL)
G. Transfers and Mobility (as appropriate)

4. The importance of a healthy lifestyle and secondary preventions:
A. Control of blood pressure
B. Use of aspirin/warfarin or similar
C. Smoking
D. Appropriate diet, including prevention of excess weight gain
E. Exercise
F. Pain management (mandatory)

5. Dietary needs and feeding techniques

A. Special diet

B. Techniques to assist eating, including use of specialist equipment if necessary (as appropriate)

6. How to communicate with dysphasic relative (as appropriate)

7. How to manage relative's personal washing, dressing, toiletry needs (as appropriate)

8. The importance of limb positioning and the management of pressure areas and skin integrity (as appropriate)

9. Continence management (as appropriate)

10. Bowel management, fluid and dietary intake for the prevention of constipation (as appropriate)

11. Appropriate techniques and ability in

A. Safe transfers

B. Safely assisting mobility

C. Floor routine following a fall

D. Safely assisting in climbing stairs

E. Good use of a wheelchair

F. Use of aids (as appropriate)

12. The importance of compliance with medication (including supervision of self-medication or routine medication; mandatory)

13. Post discharge arrangements and where and whom to seek help from after discharge (mandatory)

14. Adapting the knowledge and skills taught to the home environment following discharge (follow-up visit or phone call; mandatory)

Previously published in: Clarke et $a .^{15}$

participating in the trial; gain understanding of patients', caregivers' and staffs' experience of training and understand patients' and caregivers' perceptions of the benefits of training. We report here on the first two aims.

\section{METHODS}

\section{Settings}

A subsample of 8 SUs (4 intervention, 4 control, representing 2 units in each region) from the 18 intervention 
and 18 control sites participating in TRACS were independently selected by Clinical Trials Unit staff using a purposive sampling frame of control/intervention site, quality of care measured by performance (upper and lower quartile) in 10 domains identified in 2006 National Sentinel Stroke Audit ${ }^{10}$ and geographical site (table 1). Control units were included in the process evaluation to understand the nature and frequency of informal caregiver training that may already be routinely occurring. Participant eligibility was as used in the TRACS trial (box 2). ${ }^{6}$ Four researchers each undertook fieldwork between February 2009 and July 2009, in an intervention and control SU in each of the four regions included in TRACS. Site access was negotiated with co-investigators and clinical leads at each site. To test emerging interpretations of process evaluation findings, observations were later conducted in two additional intervention units between October and December 2009 (table 1 SUs E-F). Separate funding, NHS Research Ethical approval (Reference: 08/H1307/104) and NHS Trust approval were required for the process evaluation which started almost 12 months after the TRACS trial began recruiting. Participants provided written informed consent prior to observations and interviews.

\section{Data generation and analysis}

An ethnographic approach ${ }^{11}$ was adopted which included qualitative observations, interviews with MDT members, patients and caregivers and documentary analysis. In intervention units, we evaluated how the LSCTC was understood by staff and received by caregivers; what factors facilitated or inhibited its implementation and what caregivers perceived the effects of training to be. In control units, researchers recorded current practice including instances of caregiver training. In control and intervention SUs we observed nurses' and healthcare assistants' work between 07:00 and 21:30 h, therapy staffs' work (normally 8:30-16:30) and physicians and others if caregiver training or information provision was planned. Observations moved from general SU routines (shift handovers, discipline specific and MDT meetings), to patient and caregiver sessions in therapy spaces, kitchen, bed areas on SUs and home visits. Observations and interviews with patient-caregiver dyads were negotiated by researchers on an individual and on-going basis following patient admissions, in consultation with MDT members, typically after intention to provide caregiver training was mentioned in therapy, MDT or family meetings. The same patient-caregiver dyads were observed on repeated occasions where possible. An observational framework developed for the study (see online supplementary file 2) was used to record researchers' observations, reflections, interactions between staff and between staff and caregivers. Documentary analysis was undertaken of therapy, nursing or MDT records for the observed patient-caregiver dyads to identify recorded information (focus and duration) on caregiver training delivered; these data were summarised and compared with observational records from control and intervention sites, and with training records in intervention sites. After on-site data collection ceased we accessed intervention compliance data from training record returns.

Patient and caregiver dyads were interviewed once, at home, 3 months after discharge (May-October 2009). This time period allowed patients and caregivers to adjust to being at home, to establish daily routines and to reflect on whether information provided and skills developed in hospital had been helpful. Patients and caregivers could choose joint or separate interviews; just over $50 \%$ preferred joint interviews. Using a purposive approach we interviewed dyads who had been observed receiving LSCTC or informal caregiver training. Interview topics explored included in-hospital and postdischarge experiences, recollections and experiences of caregiver training and perceptions of benefits for life at home. Analysis of these interview data informed subsequent MDT interviews in certain areas, for example, as caregivers reported written information was often generic and did not apply well to their needs, the staff topic guide explored MDT members' perceptions of verbal and written information provided in SUs. Similarly, we explored MDT members' perceptions and expectations of the caregivers' role in LSCTC or informal training, comparing these with researchers' observational and caregiver interview reports.

Single interviews were conducted with MDT members on intervention and control SUs, after trial recruitment ceased (February-May 2010). The interview topic guide explored beliefs about caregiver training, preparation for, understanding of and engagement with the LSCTC intervention, or in control units, the nature and purpose of caregiver training provided. Purposive sampling was used to recruit experienced and inexperienced MDT members, at different grades, who had provided caregiver training. Interviews were audio-recorded and transcribed verbatim.

Data generation and analysis drew on grounded theory methods including constant comparison and progressive focusing of observations and interviews. ${ }^{12}$ Regular dialogue between research fellows (RFs) was important as fieldwork, and later interviews, occurred simultaneously in four regions. To ensure consistency in approach, a qualitative observational framework was developed and agreed with RFs prior to fieldwork (see online supplementary file 2). Examples of fieldnotes from previous studies were reviewed. As well as focusing on routine practice in SUs, the framework encouraged observations of emergent areas related to LSCTC implementation, and progressive focusing on specific aspects of training, for example, supervised caregiver practice of skills. RFs also used phone and email to discuss data generation and analysis issues. Initial coding was completed by each RF within NVivo and then reviewed jointly. RFs met every 6-8 weeks for analysis and coding of fieldnotes. This progressed to development of 
Table 1 Participating SUs-process evaluation

\begin{tabular}{llllll}
\hline Trial arm & Hospital & SU type & Stroke rehabilitation bed number 2010 & ESD scheme & Compliance (\%)* \\
\hline \multicolumn{2}{l}{ Observations } & were conducted in control units & and intervention units A-D between February and July 2009 & \\
Control A & Community & Rehabilitation & 19 & No & NA \\
Control B & Acute & Combined & 12 & No & NA \\
Control C & Acute & Combined & 12 & Yes & NA \\
Control D & Acute & Combined & 16 & Yes & NA \\
Intervention A & Acute & Combined & 20 & No & 0.0 \\
Intervention B & Acute & Rehabilitation & 18 & No & 29.6 \\
Intervention C & Community & Rehabilitation & 23 & No & 61.9 \\
Intervention D & Acute & Combined & 16 & Yes & 24.0 \\
Observations were conducted in intervention units E and F between October and December 2009 & \\
Intervention E & Acute & Combined & 23 & Yes & 43.3 \\
Intervention F & Acute & Rehabilitation & 24 & No & 77.1 \\
\hline
\end{tabular}

${ }^{*}$ Average compliance rate with completion of the training record (18 intervention sites) $=43.6 \%$.

ESD, early supported discharge; NA, not applicable; SU, stroke unit.

analytical categories and subcategories which provided a credible account of patterns in the data. The same approach was used to analyse interview data. Data generation, coding, emerging interpretations and analytical categories were discussed with steering group members every 12 weeks.

Emerging findings from observations in the four intervention SUs identified variable engagement of MDTs in LSCTC delivery. Therefore, observations, as described above, were undertaken in two additional intervention sites, one in the South East, one in Yorkshire. These examined whether the explanation of routine practice and delivery of the LSCTC developed in the first four SUs was atypical or whether this was transferable to other

\section{Box 2 Eligibility criteria process evaluation}

Patients and caregivers

Confirmed primary diagnosis of new stroke.

Medically stable.

Likely to return home with residual disability at the time of discharge.

Have a caregiver available, defined as the main person other than health, social or voluntary care provider, helping with activities of daily living and/or advocating on behalf of the patient, who is willing and able to provide support to the patient after discharge.

Patient and caregiver consent to caregiver participation in the study.

Stroke unit staff

All stroke unit team members in study sites were eligible for participation in the observational and interview components of the process evaluation, provided that they were providing medical, nursing or therapy care for patients after a new stroke on a routine and regular basis during the course of the process evaluation.

Stroke Association Family Support workers were eligible for inclusion if they were providing information and support for patients and caregivers on the stroke unit on a routine and regular basis during the course of the process evaluation. intervention SUs. Data from the additional sites supported the original explanation. As fieldwork was completed in the first eight units, we undertook a post hoc analysis of our data using Normalisation Process Theory (NPT) ${ }^{13}$ NPT is an explanatory theoretical model which focuses on how complex interventions are introduced, understood, embedded and can become 'normalised' within routine practice. NPT identifies four generative mechanisms: coherence, cognitive participation, collective action and reflexive monitoring (table 2). These mechanisms represent activities that people engage in when an intervention is introduced and implemented. Details of the research methods and use of NPT are published elsewhere. ${ }^{14} 15$

\section{RESULTS}

Over $1200 \mathrm{~h}$ of observation were undertaken. Thirty-eight patient-caregiver dyads and 53 MDT members were interviewed. Participant characteristics are summarised in table 3. We report on three factors which appeared to influence introduction and implementation of the LSCTC. These explore contextual factors including external and internal pressures which, staff recognised as being designed to improve services, but nonetheless impacted on MDT members' engagement with the LSCTC or with informal caregiver training in SUs. Related to the issue of engagement we then examine poststroke influences on caregivers which impacted on their readiness to appreciate the need for and participate in training designed to help them support stroke survivors at home.

\section{Contextual factors including organisational,} multidisciplinary and local stroke unit practice impacted on LSCTC introduction and implementation

The LSCTC was implemented in participating SUs from February 2008 to February 2010, our study identified that the stroke care context was challenging with national pressures to further develop services, for 
Table 2 Normalisation process theory: the work of implementation four inter-related generative mechanisms (after May and Finch $^{11}$ )

\section{Contexts}

The generative mechanisms are considered to be in dynamic interaction and are influenced by individual and wider, professional, local practice and organisational contexts

\begin{tabular}{|c|c|}
\hline $\begin{array}{l}\text { Generative } \\
\text { mechanisms }\end{array}$ & Explanation \\
\hline Coherence & $\begin{array}{l}\text { Coherence [individually and collectively]relates to: } \\
\text { how the work that defines and organises a practice/ } \\
\text { intervention is understood, rendered meaningful } \\
\text { and invested in, in respect of the knowledge, skills, } \\
\text { behaviours, actors and actions required to } \\
\text { implement it }\end{array}$ \\
\hline $\begin{array}{l}\text { Cognitive } \\
\text { participation }\end{array}$ & $\begin{array}{l}\text { Cognitive participation relates to: commitment to } \\
\text { and engagement of participants with the } \\
\text { intervention. Do participants view the intervention } \\
\text { as something worthwhile and appropriate to commit } \\
\text { their individual time and effort [signing up] to bring } \\
\text { about the intended outcome? }\end{array}$ \\
\hline Collective action & $\begin{array}{l}\text { Collective action relates to: the work that will be } \\
\text { required of participants to implement the } \\
\text { intervention, including preparation and/or training. } \\
\text { How far will existing work practices and the division } \\
\text { of labour have to be changed or adapted to } \\
\text { implement the intervention? Is the intervention } \\
\text { consistent with the existing norms and goals of the } \\
\text { groups, the workplace and overall organisation [this } \\
\text { is policy, practice and service user linked] }\end{array}$ \\
\hline $\begin{array}{l}\text { Reflexive } \\
\text { monitoring }\end{array}$ & $\begin{array}{l}\text { Reflexive Monitoring relates to: participants' } \\
\text { individual and collective on-going formal and } \\
\text { informal appraisal of the intervention and its } \\
\text { benefits for participants, in relation to realising } \\
\text { individual and organisational goals }\end{array}$ \\
\hline
\end{tabular}

Previously published in: Clarke et al. ${ }^{15}$

example, to introduce hyperacute services and reduce length of stay. 81416

Because of pressures of the NHS and the fact that we've got to reduce length of stay... we're getting much more pressure to set a discharge date. [Occupational Therapist (OT) intervention site B]

Therapists in particular highlighted that reducing length of stay also reduced time available for MDT members to determine the need for and provide individual LSCTC training. This quote from an experienced OT was indicative of the commonly reported pressure to reduce length of stay in all SUs.

Contextual conditions for introducing the LSCTC were more favourable (with higher intervention compliance) where team leads had been in post for some time; staffing levels were stable and where team members expressed a view that audit, research and change were central to service improvement. ${ }^{715}$

I think you need to engage with these [studies]... You can't just sit with thinking, we've always done it this way...

There has to be someone who takes that step back and looks at things and looks at outcomes as well [.....]. So, in some ways it absorbs resources but in other ways it supports development... [Speech and language therapist (SALT), intervention site B]

This participant appeared to perceive research as part of a commitment to examine the effectiveness of new approaches, such as the LSCTC, in routine practice. There is recognition of the additional demands trial participation brings but this is viewed as worthwhile as the outcome can be service improvement. This kind of positive perception of research and service improvement initiatives was not evident in all the SUs observed.

LSCTC implementation was challenging from the outset in three intervention units with high staff turnover, where the staff felt change was imposed or not relevant to their role, and where interprofessional relations were already problematic. In two of these SUs uncertainty regarding service reorganisation, including senior staff competing for posts, impacted on leadership and staff morale. Compliance data from these SUs were consistent with our observations, demonstrating little evidence of caregiver training (box 2).

The Stroke Unit Trialists' Collaboration ${ }^{17}$ identified that an MDT approach is a key element of the effectiveness of SU care. However, MDT members largely worked separately in discrete professional groups with therapy typically occurring in separate spaces, sometimes away 
Table 3 Participant characteristics

\begin{tabular}{lll}
\hline Patient & Intervention (16) & Control (22) \\
\hline Age, mean (SD) & $69(15)$ & $74(15)$ \\
Barthel score at discharge, mean (SD) & $12.3(5)$ & $9.8(5)$ \\
Number of females (\%) & $6(38)$ & $14(64)$ \\
Number with language impairment (dysphasia) (\%) & $7(32)$ \\
Caregivers & $5(31)$ & Control (22) \\
Age, mean (SD) & Intervention (16) & $67(13.9)$ \\
Number of females (\%) & $59.9(13)$ & $11(52)$ \\
Staff & $11(69)$ & Control (30) \\
OT & Intervention (23) & 6 \\
Physiotherapist & 5 & 7 \\
SALT & 5 & 2 \\
Nurse & 2 & 9 \\
Healthcare assistants & 8 & 1 \\
Medical staff & 1 & 1 \\
Stroke association family support workers & 0 & 2 \\
Dietician & 2 & 0 \\
Social worker & 1 & 1 \\
Previously published in: Clarke et al. ${ }^{15}$ & 0 & \\
OT, occupational therapist. & &
\end{tabular}

from SUs. The work of MDT members was therefore not always visible to their colleagues and we observed only limited evidence of collective action in LSCTC implementation.

Because we're focussing on getting people admitted, doing the initial interview, the baseline assessments and finding out what problems they've got, then we've got very little time to actually do the rehab programmes and work with the person on those activities. [Occupational Therapist [OT], intervention unit A]

This OT indicates some of the reasons why MDT members concentrated primarily on ensuring their own patient assessments and documentation were completed. Working separately is a common strategy to complete work required but in some SUs it led to assumptions being made that other MDT members would cover, or had covered LSCTC elements. Caregiver training was not routinely reported on in MDT or other meetings in intervention or control units. Separate working practices, separate care records and infrequently consulted shared MDT records limited opportunities for information exchange between disciplines about caregiver training. As treating the patient was the primary focus for MDT members, caregivers' training needs often appeared of secondary concern until a discharge date was near; training then assumed a higher priority for some MDT members.

However, some aspects of work organisation in SUs limited the availability of caregivers to participate in training. The majority of therapy or care took place in mornings or early afternoons, whereas most caregivers were present in afternoons and evenings. Initiatives designed to improve care, including protected mealtimes and restricted visiting hours, largely served to exclude caregivers. Open visiting provided training opportunities but these were rarely utilised. Sometimes staff reported it was inappropriate to use visiting time to train caregivers, on other occasions they chose to exclude caregivers when therapy or care took place during visiting:

[It] can be quite a challenge for us [seeing caregivers during visiting] because that's also therapy time, so we can find that we're getting caught by quite a lot of relatives who want to ask us questions [...] in between half two when the visiting starts and 4 o'clock when we normally finish. [Physiotherapist (PT), intervention unit A]

Some caregivers reported that visiting time should be for conversation and not for learning how to care for their relative. In the next section we report on how the staff were prepared to use the LSCTC in intervention SUs and how this appeared to influence its provision.

\section{Staff engagement with the LSCTC and caregiver training}

Before the TRACS trial, experienced therapists and nurses from intervention units were trained in regional workshops, by staff that developed the LSCTC, to act as TRACS champions and 'cascade' training in their SUs. Cascade training described the LSCTC components and explored how these could be delivered locally. Cascade training was not effective in engaging all MDT members. Pressures on length of stay identified above were not reported to have impacted on participation in cascade training. However, other factors were perceived to impact. In two units, staff who attended workshops left soon afterwards without training colleagues. Staff interviewed reported cascade training was largely therapist led, attended mainly by therapists and did not reach all nurses. The unpredictable nature of nurses' work 
and continuous demand for nursing care was perceived to contribute to fewer nurses attending training, which was typically a brief one-off event, not repeated during the trial. Use of supplementary LSCTC training tools, a DVD and manual, was not observed in any unit during the process evaluation.

At interview the majority of intervention unit staff, from all professional groups, argued that caregiver training was already part of their practice and the LSCTC only made explicit the requirement to record this:

TRACS is what we should be doing anyway, it's just to formalise ... the check list and to make sure that we do feel that everything is fully covered which we've found quite useful. [PT intervention unit D]

Observations did not provide evidence to support these claims. Engagement, particularly of experienced therapists, with selected LSCTC elements was evident in all intervention sites observed. Less experienced therapists on training rotations were focused on providing therapy and less aware of the LSCTC. Nurses acknowledged caregiver training was important but most perceived it to be a therapy activity and not something that could be incorporated into their routine work:

Because the therapists went to the original training session and they would lead on whether they decided a patient was for TRACS or not, it was very therapy based. The nurses and myself really, didn't really take ownership of it. [Nurse Specialist intervention unit D]

Medications, observations and it's the acute care side of things, and all the basic washing and getting them up. That takes all day, basically, there's not a lot of time for anything else. [Nurse Specialist intervention unit C]

One mandatory LSCTC element, verbal and written information provision about stroke by members of the MDT, was regularly observed in all units. However difficulties in information retention by caregivers were recognised:

I guess part of it is to do with how people deal with shock [...], so you can tell someone....they can hear the same information from three different people and the next day they will still struggle to recall it or they will still feel that they haven't been given that information. [SALT Intervention unit C]

Researchers frequently observed information on stroke, related impairments and disabilities being provided by doctors, nurses and, in four SUs, by Stroke Association Support Workers. Patients and caregivers reported variable recall of this information but were much more consistent in reporting that written information, often provided in standard booklets, was not specific enough to meet their needs and largely proved unhelpful to them after discharge.
Assessment of caregiver competency was also challenging

Some of the problems that we found with it [...] it was partly I think looking at competence, not just physically giving out a leaflet to people, it [TRACS] was going beyond that wasn't it really [....] but I think [...] even after a lot of training, people aren't necessarily competent communication partners for example, which is fair enough, it's a real skill, it takes a long, long time to develop. So from my point of view I found it quite difficult that, although I could sign off that I'd given them the information for example and have spent time discussing it, I think there was probably nobody that actually I could say was competent having had that information, and I wouldn't expect them to be. [SALT, intervention unit A]

We found little evidence that the complexity identified by this SALT in developing caregiver competence was part of cascade training or discussed by SU staff. Few instances of caregiver competency assessment were observed in intervention SUs.

Risk management concerns also strongly influenced content and timing of caregiver training. Therapists frequently stated it was important to delay training until patients' likely functional recovery potential was known and caregiver skills required postdischarge could be determined. This resulted in most training being provided in the days prior to, or the week of discharge and in limiting training to reducing perceived risks, for example, fall risk or safe management of swallowing.

Therapists reported home visits were used to provide training where it was most useful to caregivers. We observed only a few home visits so cannot verify these claims; those we did observe typically involved information giving rather than skills training and thus continued the in-hospital pattern of caregiver instruction. Except where early supported discharge (ESD) schemes were in place (box 2) no caregiver training equivalent to the LSCTC was evident in process evaluation study sites.

\section{Influences on caregivers poststroke}

Recall of training was very limited even for those we had observed receiving some LSCTC training. Caregivers' recall of information provision varied but a common perception (differing from observations) was that while much written information had been provided, it was repetitive, generic and not focused on their individual needs. Perceptions were influenced by the 'shock' associated with stroke as noted in other studies. ${ }^{1}{ }^{18-20}$

You think 'it's not real this, is it? It's a dream, isn't it, we haven't woken up from it yet', this isn't really happening to us, is it? [Caregiver, control unit A]

Willingness to participate in training was partly influenced by pre-existing relationships between patients and caregivers and partly by perceptions of social obligations 
and prior caring experiences. Those who had prior experience of caring roles and those who perceived they had a moral obligation to care for a partner, which they expected would be reciprocated, were unlikely to question being ascribed the role of caregiver. Most accepted or were willing to assume the role but some resisted and some were unwilling to learn specific skills such as tube feeding and intimate personal care.

Some caregivers perceived training to be unplanned; they were uncertain what was expected of them in a caregiving role:

It was just if you happened to be there. I saw about three or four [therapy sessions] I think, so I did see what they were doing but none of them said, "When he's home try and make him do this or try and make him do that". [...] it was just a case of watching and seeing what they were doing and how they did it. [...] but no, they never said "You do this or you do that. [Caregiver, intervention unit C]

This was consistent with many observations in intervention and control SUs. Caregiver training sessions were often planned but many were opportunistic and depended on whether caregivers were visiting on a particular day. We observed, as suggested in the quotation, the passive role adopted by most caregivers when attending training with MDT members at SUs. Staff appeared to assume caregivers would develop skills by simply watching therapy being provided. Exceptions were observed with deliberate active caregiver involvement, for example, in learning how to help stroke survivors after a fall, or to thicken fluids and support safe swallowing. However, over half of caregivers interviewed reported the skills they developed in moving and handling, transfers, hoist use and personal care resulted from repeated observations of social care staff or community therapists rather than a planned sequence of demonstration, practice and assessment of competence in LSCTC elements:

When the occupational therapist brought her back home and I saw her and watched her do everything with my mum and I really was making [notes]. [Caregiver, Control unit B]

Recall of training was more evident where ESD schemes operated and where therapists focused on specific skills or information perceived as important by patients and caregivers in the home.

\section{DISCUSSION}

The TRACS trial aimed to enhance patients' functional recovery, and reduce caregivers' burden by providing an in-patient, structured caregiver training programme, the LSCTC. The TRACS trial did not demonstrate any benefit of this intervention for patients or caregivers. ${ }^{6} 7$ The process evaluation provides insight into these findings. Senior therapists in most intervention units developed ownership of the LSCTC, but this was not evident across MDTs. Preparatory cascade training on delivery of the LSCTC did not reach all staff and did not lead to MDT-wide understanding of, engagement with or commitment to the LSCTC. With the exception of some ward sisters, most nurses did not know about, or had little understanding of the LSCTC. This was also true of some therapists on training rotations and some physicians. Organisational features of SUs and professionals' working practices also limited the involvement of caregivers. MDT working contributed to separation rather than integration of delivery of LSCTC elements. As a result only some competencies were regularly addressed. Skills training and assessment of caregiver competence was not commonly observed (or recalled by caregivers), where it did occur it was mostly delivered by experienced therapists. Caregivers were often invited to observe therapy or care being provided but had few practical opportunities to develop skills in the $\mathrm{SU}$ context. This made it difficult to make sense of and commit to developing knowledge and stroke-specific skills provided in the LSCTC. MDT and family meetings occurred in all units but rarely focused on caregiver training, thus no routine forum existed for staff to review and monitor caregiver training during TRACS.

Caregivers' desire for information and skills training is well established..$^{2} 3$ 18-21 22 In TRACS, during patients' hospitalisation most caregivers were still coming to terms with the shock of their relatives' stroke and often unaware of the demands that would be made upon them on their return home; features reported in other studies. ${ }^{1} 19{ }^{20} \mathrm{SU}$ staff assessed caregiver readiness for and understanding of LSCTC training on an individual basis but did not always make explicit that training was designed to provide skills for coping at home, and were rarely able to provide time and opportunity for skills practice. Where provided, caregiver training came very late in the inpatient stay. The most appropriate time to provide caregiver training after stroke has not been definitively established. There is evidence that caregivers' needs in terms of training and support are subject to change over time. Therefore, training limited to inpatient or community settings alone may not address needs arising at differing time points. ${ }^{18-20} 23$ The SU-based LSCTC, and caregiver training in control units were not reported as occurring too soon but had limited impact on caregivers' perceptions of their capacity to provide patient support. The use of 'muddling through' by observing how things were carried out by carers or community staff, seeking help from family or friends and finding their own solutions to challenges at home was reported and has been noted in other studies. ${ }^{19}{ }^{20}$ With the exception of those participating in ESD schemes, no direct benefit from training was reported by caregivers. In our view, the timing of the LSCTC intervention was a factor in why TRACS found no difference in clinical or statistical outcomes between intervention and control groups. However, evidence is 
still required to establish what caregivers and professionals regard as effective training to support patients after discharge, when training should be delivered and what kind of programmes are feasible to deliver within the existing resources of stroke services. Although TRACS did not result in improved outcomes for patients or caregivers, ${ }^{6}{ }^{7}$ we found no evidence that the LSCTC was regarded as an inappropriate caregiver training model. Moreover, we found no evidence that caregivers rejected or perceived that training (where offered) was not relevant to their needs. Some caregivers wanted more training, although a small number resisted specific skills training where it conflicted with established kinship roles.

One possible explanation for the main TRACS trial outcome is that participating SUs were already providing LSCTC like caregiver training. This explanation was not supported by observational data. Nonetheless, many MDT members reported that caregiver training was already occurring. This perception may explain why intervention unit staff did not all engage with and deliver the LSCTC. Similar perceptions were evident in control units. Despite preparing intervention unit staff through workshops and cascade training, TRACS could not replicate the motivation, commitment and control over delivery of LSCTC evident in the Kalra $e t a l^{3}$ study where the same staff were responsible for delivery and where intervention fidelity was assured. Intervention SUs had similar organisational characteristics to those in the single centre trial. ${ }^{3}$ TRACS was a pragmatic trial designed to establish if the LSCTC was effective under routine practice conditions in a range of SUs. Intervention unit staff were not persuaded that delivery of all elements of the structured LSCTC programme, effective in the single centre trial $^{3}$ would be more effective than their current unstructured approach.

LSCTC implementation is unlikely to be practical in settings with short inpatient stays. Stroke services continue to change, with improvements in recognition and treatment of stroke in the acute phase and reductions in length of stay as ESD and other community rehabilitation services become established. Wherever caregiver training is provided, more consideration needs to be given to processes of skills training and competency assessment which are not simple matters of demonstration and repetition. In SUs these are influenced by the requirement to manage risk, by caregivers' knowledge, attitudes, prior experience, time and practice opportunities. SUs with ESD schemes in place potentially offer a vehicle for introducing structured caregiver training consistent with the LSCTC.

The process evaluation is one of the largest conducted alongside a stroke rehabilitation trial, with over $1200 \mathrm{~h}$ of observation and 92 interviews. A major strength of the study was in generating rich and detailed findings which are important, not just in terms of interpreting the TRACS trial results but also in understanding factors influencing introduction, implementation and embedding of complex interventions such as caregiver training into these and similar settings. Obtaining separate funding, ethical and NHS approvals meant SU observations began almost 1 year after TRACS recruitment started. Observations of early implementation strategies and staff responses to these were therefore not possible, so analysis drew on interview data for this period. Another limitation of the study was that only 6 of 18 intervention, and four control SUs were observed. These factors impact on the transferability of the findings to other SUs. However, it is important to note that the grounded theory methods and the NPT approach adopted are aimed at theoretical rather than statistical generalisability. The SUs observed met the currently adopted key characteristics of SUs. ${ }^{21}$ Site selection was determined by theoretical sampling criteria and rigorous quality measures were adhered to in data generation and analysis.

The process evaluation identified that the effectiveness of the LSCTC cannot be judged simply in terms of limitations in the implementation process or intervention fidelity. Using NPT's constructs within the process evaluation helped identify vulnerable features of the implementation process with respect to the work involved in introducing and embedding the LSCTC and the importance and influence of contextual factors. ${ }^{15}$ Implementation of complex interventions such as the LSCTC into complex clinical settings will benefit from pretrial exploration of factors which may facilitate or hinder implementation. ${ }^{9} 132425$ Researchers planning to test the effectiveness of interventions such as the LSCTC should consider using a theory driven approach to implementing and embedding practice change which draws on evidence from large organisational change projects $^{132425}$ and the emerging evidence on post-stroke caregiver training programmes. ${ }^{7} 1823$ Providing time and opportunity for all MDT members to review the strengths and weaknesses of their existing practice at a local level is likely to be fundamental to engaging them in new ways of working. ${ }^{24} 25$

\section{Author affiliations}

${ }^{1}$ Bradford Teaching Hospitals NHS Trust and University of Leeds, Bradford, UK

${ }^{2}$ Leeds Institute of Health Sciences, University of Leeds, Leeds, UK

${ }^{3}$ Department of Primary Care and Public Health Sciences, King's College

London, London, UK

${ }^{4}$ Peninsula College of Medicine and Dentistry, Exeter, Devon, UK

${ }^{5}$ Clinical Trials Research Unit, Health Sciences Division, University of Leeds, Leeds, UK

'Department of Stroke Medicine Guy's, King's \& St Thomas' School of Medicine, London, UK

${ }^{7}$ Kent Community Health NHS Trust, Kent, UK

Acknowledgements The Process Evaluation team are very grateful to the patients, caregivers and staff who participated in the study. The authors also thank many people who assisted in the set up and operation of the study including the UK Stroke Research Network staff. Participating Stroke Units were located in: Kings College Hospital NHS Foundation Trust, Dartford \& Gravesham NHS Trust, East Kent NHS Foundation Trust, The Mid-Yorkshire NHS Trust, Bradford Teaching Hospitals NHS Foundation Trust, The 
Rotherham NHS Foundation Trust, Salford Royal NHS Foundation Trust, Southport \& Ormskirk NHS Trust, Cornwall and Isles of Scilly Primary Care Trust, Plymouth Teaching Primary Care Trust.

Contributors AF, CM, JD, DJC, MG, LK, DS and AJF designed the process evaluation study. DJC, RH, ES and GH collected and analysed the data. DJC developed the manuscript. RH, ES, GH, AF, MG, CM, JD and AJF reviewed the manuscript and made recommendations for changes and additions.

Funding This paper presents independent research funded by the National Institute for Health Research (NIHR) under its Research for Patient Benefit (RfPB) Programme (Grant Reference Number PB-PG-0407-13308). The views expressed are those of the author(s) and not necessarily those of the NHS, the NIHR or the Department of Health.

Competing interests None.

Ethics approval Leeds West Research Ethics Committee (Reference number:08/H1307/104).

Provenance and peer review Not commissioned; externally peer reviewed.

Data sharing statement No additional data are available.

Open Access This is an Open Access article distributed in accordance with the Creative Commons Attribution Non Commercial (CC BY-NC 3.0) license, which permits others to distribute, remix, adapt, build upon this work noncommercially, and license their derivative works on different terms, provided the original work is properly cited and the use is non-commercial. See: http:// creativecommons.org/licenses/by-nc/3.0/

\section{REFERENCES}

1. Alaszewski A, Alaszewski H, Potter J. Risk, uncertainty and life threatening trauma: analysing's stroke survivors' accounts of life after stroke. Forum 2006;7:18, [Online]. http://nbnresolving.de/urn:nbn: de:0114-fqs0601189

2. Forster A, Brown L, Smith J, et al. Information provision for stroke patients and their caregivers. Cochrane Database Syst Rev 2012; (11):CD001919.

3. Kalra L, Evans A, Perez I, et al. Training care givers of stroke patients: randomised controlled trial. BMJ 2004;328:1099-101.

4. Patel A, Knapp M, Evans A, et al. Training care givers of stroke patients: economic evaluation. BMJ 2004;328:1102.

5. Forster A, Young J, Nixon J, et al. A cluster randomized controlled trial of a structured training programme for caregivers of inpatients after stroke (TRACS). Int J Stroke 2012;7:94-9.

6. Forster A, Dickerson J, Young J, et al. on behalf of the TRACS Trial Collaboration. A structured training programme for caregivers of inpatients after stroke (TRACS): a cluster randomised controlled trial and cost-effectiveness analysis. Lancet 382:2069-76.

7. Forster A, Dickerson J, Young J, et al. A cluster randomised controlled trial and cost-effectiveness analysis of a structured training programme for caregivers of in-patients after stroke (TRACS). Health Technol Assess 2013;17:46.

8. Medical Research Council. Developing and evaluating complex interventions: new guidance. London: MRC, 2008.

9. Oakley A, Strange V, Bonell C, et al. the RIPPLE Study Team 2006. Process evaluation in randomised controlled trials of complex interventions. BMJ 2006;332:413-16.

10. Royal College of Physicians. Summary report on the National Sentinel Stroke Audit. London: Royal College of Physicians, 2006.

11. Hammersley M, Atkinson P. Ethnography: principles in practice. 3rd edn. London: Routledge, 2007.

12. Corbin J, Strauss AL. Basics of qualitative research. 3rd edn. London: Sage, 2008.

13. May C, Finch T. Implementing, embedding, and integrating practices: an outline of normalization process theory. Sociology 2009;43:535-54.

14. Clarke DJ, Hawkins R, Sadler E, et al. Interdisciplinary health research: perspectives from a process evaluation research team. Qual Prim Care 2012;20:179-89.

15. Clarke D, Godfrey M, Hawkins R, et al. Implementing a training intervention to support caregivers after stroke: a process evaluation examining the initiation and embedding of programme change. Implement Sci 2013;8:96.

16. Department of Health. National stroke strategy. London: Department of Health, 2007.

17. Stroke Unit Trialists' Collaboration. Organised inpatient (stroke unit) care for stroke. Cochrane Database Syst Rev 2013;(9): CD000197.

18. Cameron JI, Gignac MAM. Timing it right: a conceptual framework for addressing the support needs of family caregivers of stroke survivors: from the hospital to the home. Patient Educ Couns 2008;70:305-14.

19. Greenwood N, Mackenzie A, Wilson N, et al. Managing uncertainty after stroke: a qualitative study of the experiences of established and new informal carers in the first 3 months after discharge. Int $J$ Nurs Studies 2009;46:1122-33.

20. Ellis-Hill C, Robison J, Wiles R, et al. Going home to get on with life: patients and carers experiences of being discharged from hospital following a stroke. Disabil Rehabil 2009;31:61-72.

21. Royal College of Physicians. Summary report on the National Sentinel Stroke Audit. London: Royal College of Physicians, 2012.

22. Brereton L, Carroll C, Barnston S. Interventions for adult family caregivers of survivors of stroke: a systematic review. Clin Rehabil 2007;21:867-84.

23. Cameron JI, Naglie G, Silver F, et al. Stroke family caregivers' support needs change across the care continuum: a qualitative study using the timing it right framework. Disabil Rehabil 2013;35:315-24.

24. Middleton S, McElduff $P$, Ward J, et al.; on behalf of the QASC Trialists Group. Implementation of evidence-based treatment protocols to manage fever, hyperglycaemia, and swallowing dysfunction in acute stroke (QASC): a cluster randomised controlled trial. Lancet 2011;378:1699-706.

25. Greenhalgh T, Robert G, McFarlane F, et al. Diffusion of innovations in service organisations: systematic review and recommendations. Milbank Q 2004;82:581-629. 


\section{Correction}

Clarke DJ, Hawkins R, Sadler E, et al. Introducing structured caregiver training in stroke care: findings from the TRACS process evaluation study. BMJ Open 2014;4:e004473.

In the 'Strengths and limitations of this study' box (bullet point 3, line 3) it should not read ' $12 \mathrm{~h}$.' The corrected sentence is:

The process evaluation is one of the largest conducted alongside a stroke rehabilitation trial, with over $1200 \mathrm{~h}$ of observation and 92 interviews.

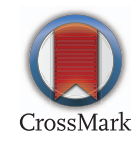

BMJ Open 2014;4:e004473. doi:10.1136/bmjopen-2014-004473corr1 